A N N A L E S Annales de Bretagne et des Pays de l'Ouest

Anjou. Maine. Poitou-Charente. Touraine

$111-4 \mid 2004$

Varia

\title{
Identité bretonne et République dans la première moitié du XXe siècle
}

Roger Dupuy

\section{OpenEdition \\ Journals}

Édition électronique

URL : http://journals.openedition.org/abpo/1179

DOI : $10.4000 / a b p o .1179$

ISBN : 978-2-7535-1496-6

ISSN : 2108-6443

Éditeur

Presses universitaires de Rennes

\section{Édition imprimée}

Date de publication : 20 décembre 2004

Pagination : $97-102$

ISBN : 978-2-7535-0082-2

ISSN : 0399-0826

Référence électronique

Roger Dupuy, "Identité bretonne et République dans la première moitié du XXe siècle ", Annales de Bretagne et des Pays de l'Ouest [En ligne], 111-4 | 2004, mis en ligne le 20 décembre 2006, consulté le 30 avril 2019. URL : http://journals.openedition.org/abpo/1179 ; DOI : 10.4000/abpo.1179 


\title{
Identité bretonne et République dans la première moitié du $\mathrm{Xx}^{\mathrm{e}}$ siècle ${ }^{1}$
}

\author{
Roger DuPuY \\ Professeur émérite \\ CRHISCO - Université Rennes 2 Haute-Bretagne
}

\begin{abstract}
À la charnière du XIX ${ }^{\mathrm{e}}$ et du XX ${ }^{\mathrm{e}}$ siècle, la séparation de l'Église et de l'État en 1905 est mal vécue par les catholiques bretons. Beaucoup de prêtres, dans le Léon (nord-ouest de la péninsule) et dans le Morbihan affirment qu'on en est revenu à 1793 et que l'anticléricalisme virulent de la République franc-maçonne veut éradiquer le catholicisme. Une fois de plus les Bretons doivent se mobiliser pour défendre leur foi et leur langue, les deux étant intimement mêlées et menacées par le jacobinisme forcené du parti radical et l'athéisme des socialistes auxquels l'anticléricalisme seul permet de justifier une alliance tactique avec un parti bourgeois. Mais les responsables politiques catholiques et l'Église préfèrent mener leur combat sur le plan national plutôt que d'encourager un quelconque séparatisme breton, car la droite est désormais nationale, sinon nationaliste, et elle ne saurait encourager une aspiration identitaire extrême. Seule une minorité de notables et d'aristocrates locaux stimulés par l'exemple irlandais, revendique un home rule persuadés que les Bretons, avec leur langue, leur folklore, leur mode de vie particulier, constituaient une Nation à part et que la reconnaissance d'une simple originalité régionale à l'intérieur de l'hexagone ne saurait leur suffire.

C'est en août 1898, qu'Anatole Le Braz, collecteur de chansons populaires, mais aussi auteur d'un très beau livre sur le sentiment de la mort dans l'art et la littérature bretonne, a fondé à Morlaix (Finistère), c'est-àdire dans la Bretagne qui parle breton, l'Union régionaliste bretonne à laquelle le marquis de L'Estourbillon, un noble haut breton du pays de Redon qui a appris le breton et qui s'est fait élire député à Vannes, va donner une dimension politique en revendiquant le droit de parler et donc d'en-

1. Cette communication est destinée à faire le point pour nos collègues italiens des acquits de l'historiographie des trente dernières années concernant l'histoire contemporaine de la Bretagne dans la perspective de notre séminaire. Elle a été essentiellement réalisée à partir des ouvrages des éditions Privat, Bourdessoule, Ouest-France Université et Skoll Vreizh.
\end{abstract}


seigner librement le breton. À l'ancien binôme foi catholique et langue bretonne on substitue une vision plus politique exaltant le peuple breton qui doit sauver sa langue par des aménagements institutionnels, car le dévouement des militants n'y suffit plus et aussi parce que l'Église glisse insensiblement vers l'usage permanent du français.

En 1911, une étape nouvelle est franchie, par la création à Rennes, capitale de la province située dans sa moitié non bretonnante, du Parti nationaliste breton, qui ne limite plus son programme à la seule défense de la langue, mais revendique la séparation comme objectif politique ultime et légitime.

Il s'agit toujours d'une minorité de quelques centaines d'individus, des notables amoureux de leur langue et passionnés de littérature et d'histoire. La masse des locuteurs bretons ne s'inquiètent guère du destin de leur langue et ne s'opposent pas à la francisation méthodique imposée par l'école primaire de la République, mais aussi par les établissements scolaires catholiques qui jouent également la carte de la francisation, synonyme de promotion culturelle, d'accès à la fonction publique, de participation à l'essor de certains secteurs économiques (marine marchande, hôtellerie), et favorisant l'émigration vers la ville, c'est-à-dire surtout vers Paris.

À la veille de la guerre de 1914-1918, deux clichés s'opposent, deux mythes, chacun ne prenant en charge qu'une partie de la réalité humaine de la péninsule. La Bretagne des régionalistes et, a fortiori, des séparatistes, est surtout celle de l'intérieur, l'Arcoat ou " pays de la forêt ", celle des paysans qui ont su conserver leurs coutumes, c'est-à-dire surtout leur foi et leur langue, celle qu'il faut défendre contre les séductions, mais aussi contre les empiétements d'une République athée, frelatée, corruptrice, uniquement convaincue de l'excellence culturelle de la capitale et pleine d'une condescendance arrogante à l'encontre d'une culture de paysans arriérés manipulés par leurs prêtres et quelques nobliaux d'un autre âge.

À cette Bretagne archaïsante, l'opinion française préfère celle de l'Armor ou " pays de la mer ", à la fois sauvage et généreuse, ouverte sur les grands horizons, la Bretagne des grands ports du commerce (Nantes, Lorient, Saint-Malo) et des petits ports de pêche pittoresques, celle des bains de mer et du voyage outre-mer. La littérature (Loti, Hector Malot, Tristan Corbière), la peinture (Boudin, une partie de l'École de Pont-Aven), la musique contribuent à célébrer ce primat de l'Océan. Dans cette perspective, l'Argoat ne serait que le prolongement occidental d'un Ouest armoricain qui commence en bordure du Bassin parisien pour mourir aux abords de Châteaulin et de Brest, avec le sentiment que pauvreté du sol et misère s'accroissaient à mesure que l'on s'éloignait de la région parisienne et qu'il y avait là un bastion résiduel de l'obscurantisme religieux et de la réaction politique.

Ces deux stéréotypes masquaient finalement la triple réalité de l'action convergente de l'école primaire publique ou confessionnelle prolongée par la presse locale et nationale, du service militaire et de la migration vers Paris. 
Désormais, une large partie de la population adhère aux institutions de la République, à un régime qui a tenu ses promesses d'ordre intérieur, d'un enrichissement modeste mais effectif du monde paysan, tandis que l'École et l'émigration multipliaient les chances d'une amorce d'ascension sociale via les niveaux élémentaires de la fonction publique, les grades subalternes de la marine ou de l'armée, la patience obstinée du petit commerce.

La guerre de 1914-1918 le confirme tragiquement : 120000 tués, ce sont environ $10 \%$ des mobilisés bretons qui ne reviendront pas, contre 7 à $8 \%$ dans la moyenne des départements français. C'est dire l'abnégation résignée de ces unités durement mises à contribution par le commandement. C'est aussi constater que les Bretons ont accepté de mourir pour défendre le territoire de la République et récupérer l'Alsace-Lorraine, c'est-à-dire qu'ils ont accepté la logique des frontières naturelles ce qui implique l'intégration de la Bretagne dans l'entité territoriale française. Finalement, le 11 novembre sera désormais célébré en Bretagne de façon plus intense que le 14 juillet, car cette commémoration prolongeait celle, traditionnelle, du souvenir des défunts lors de la Toussaint et du jour des morts et s'inscrivait logiquement dans le climat funèbre du mois de novembre que les Bretons appellent le miz $d u$, le mois noir.

C'est aussi que la " grande guerre " fut pour la Bretagne, à l'écart du front et portion importante de notre façade maritime, un moment d'intense stimulation économique. Une partie des chantiers navals fut reconvertie en usines d'armement qui tournèrent intensément de 1915 à 1919. La chaussure à Fougères, connut un essor spectaculaire. Brest devint le port d'exportation de matériel de guerre vers la Russie de Nicolas II via Mourmansk et Arkhangelsk, soit 500000 tonnes de 1914 à 1917. Et la capitulation des Russes est compensée par l'arrivée massive des Américains qui vont faire de Brest et de l'estuaire de la Loire, les principales bases des forces américaines. À cela s'ajoute l'essor de l'industrie alimentaire, conserveries et biscuiteries, surtout à Nantes. La Bretagne était devenue un des lieux majeurs où se manifestait, de façon spectaculaire, l'effort de guerre des alliés.

Et cette euphorie perdure aux lendemains immédiats du conflit. Les importations de charbon britannique prennent le relais du matériel de guerre et la métallurgie se développe sur certains sites portuaires (Nantes, Saint-Nazaire) avec, en prolongement, l'essor de la construction mécanique (locomotives et automobiles, toujours à Nantes). Des ministres de la marine bretons lancent des programmes de modernisation des ports et de leurs flottes de pêche. La politique d'électrification en milieu rural entraîne la construction de barrages, notamment dans le centre de la Bretagne intérieure (barrage de Guerlédan).

Mais la crise de 1929 casse brutalement cette dynamique. Aux conditions globales de la crise dont on sait qu'elle se déclenche en France avec deux ans de retard, s'ajoutent des difficultés particulières pour l'économie bretonne. La remise en marche des houillères du Nord provoque l'arrêt progressif des importations de charbon anglais et aussi, en manière de 
rétorsion, des exportations de poteaux de mine et de légumes vers la Grande Bretagne. Le Havre supplante Saint-Nazaire comme tête de ligne des liaisons vers les États-Unis et les Antilles. Les prix de la sardine en boîte s'effondre face à la concurrence espagnole qu'on laisse jouer pour pouvoir exporter des voitures outre Pyrénées. L'exploitation des mines de fer locales est abandonnée entraînant la fermeture de plusieurs forges, témoins du passé sidérurgique de la province.

Toutes ces difficultés s'additionnent et créent un malaise que la minorité des militants nationalistes bretons pensent pouvoir exploiter. Leur presse dénonce l'égoïsme des industriels, des " charbonniers " et des vignerons français qui imposent leurs intérêts aux dépens de ceux de la Bretagne.

Alors que dès 1919, de L'Estourbillon s'adresse à la SDN et au Président Wilson pour obtenir le droit à l'existence de la langue bretonne, tandis que d'autres militants lui demandent d'exiger de la France un Home rule pour la Bretagne à l'image de ce que les indépendantistes irlandais ont exigé de Londres, de jeunes lecteurs de Maurras trouvent que l'Union Régionaliste Bretonne s'enlise dans un modérantisme sans issue et regardent, fascinés, l'aventure mussolinienne en Italie. Parmi eux, Olivier Mordrel, se sent l'étoffe d'un chef et prône une stratégie offensive d'intimidation en vue d'imposer à une France en crise et donc en déclin, la création d'un État breton autonome. Un journal bimensuel, bilingue, intitulé Breiz Atao, qui signifie "Bretagne debout! " commence à diffuser leurs mots d'ordre : la Bretagne doit devenir un État et il faut donc en finir avec le joug imposé par la France et donc se donner les moyens d'un coup de force au moment propice.

En 1927, Mordrel et une poignée d'amis (François Debauvais, Morvan Marchal qui invente le drapeau breton, blanc et noir - le gwenn ha du-, Duhamel) créent le Parti autonomiste breton. Dès septembre 1927, se tient, à Quimper un colloque regroupant des autonomistes Alsaciens, Corses et Flamands. Mais la crise entraîne l'accroissement brutal des difficultés financières de nos autonomistes et le départ de la composante de gauche du parti derrière Duhamel, laissant à Mordrel et Debauvais l'opportunité, avec tous les autres militants et sympathisants, de créer, en 1931, le Parti National Breton d'inspiration notoirement fascisante.

Le 7 août 1932, le PNB commémore, à sa façon, le quatrième centenaire du Traité d'union entre le royaume de France et la Bretagne, en dynamitant le groupe de statues qui, dans une niche, sur la façade de l'Hôtel de Ville de Rennes, magnifiait l'événement et qu'on avait inauguré en 1911. Au mois de novembre de cette même année, la voie ferrée de Nantes à Rennes par laquelle doit arriver dans la capitale bretonne, le Président du conseil, Édouard Herriot, est sabotée.

Ces deux attentats provoquent une répression policière qui cherche à démanteler le mouvement : son journal est interdit, plusieurs militants arrêtés. L'opinion publique, en général, dénonce ces procédés, mais le parti communiste soutient ce qu'il estime être un combat légitime. À partir de 
1938, la dégradation des relations entre la France et l'Allemagne hitlérienne, incite Mordrel et Debauvais à entrer en contact avec les nazis pour exploiter au mieux les pressions exercées par Berlin. L'exemple irlandais les persuade de l'efficacité et donc de la légitimité de cette stratégie. Le PNB s'est doté d'une formation paramilitaire que les Allemands arment clandestinement (août 1939) tandis que nos deux leaders quittent l'hexagone pour se réfugier outre-Rhin.

Mais ces choix politiques radicaux ne mobilisent tout au plus qu'un gros millier de sympathisants et quelques centaines de militants convaincus, car, de son côté, la République relance ses investissements dans la péninsule. L'amiral Darlan impose ses objectifs aux politiques et obtient un effort budgétaire qui permet, à partir de 1937, une montée en puissance de la construction navale militaire à Brest, Saint-Nazaire, Nantes et Lorient : deux cuirassés ultra modernes (Richelieu et Jean Bart), des torpilleurs et des sous-marins sont mis en chantier. La construction civile connaît également un nouvel essor lié au développement du mythe impérial, de l'intensification des échanges avec l'Afrique du nord et de la compétition internationale transatlantique : lancement du Normandie mais aussi d'une dizaine d'autres paquebots, construction de pétroliers, de bananiers et de cargos plus modestes voués au cabotage colonial, modernisation de la flotte de pêche et des installations portuaires pour la valoriser, essor spectaculaire des raffineries de pétrole (Donge). Il faut y ajouter une usine d'armement à Nantes, et l'expansion de l'industrie aéronautique tant Nantes qu'à Saint-Nazaire.

Paradoxalement, les menaces qui pèsent sur la France à partir de 1937, profitent à l'économie bretonne et donc enlèvent à l'argumentation séparatiste une large part de sa force de persuasion. La renaissance des ambitions maritimes de la République, le mythe d'un empire colonial présent sur les cinq continents et que la France doit mettre en valeur, la nécessité de développer des industries d'armements à l'abri des bombardements de l'Axe germano-italien, le développement des échanges avec les Antilles et l'Amérique du Nord sont autant de facteurs qui valorisent la position stratégique de la Bretagne et le savoir-faire de ses populations. Ajoutons que la marine nationale et l'armée restent des débouchés traditionnels pour la population masculine des cinq départements bretons, et donc, là encore, la tension internationale accroît des besoins déjà stimulés par le développement de la marine de guerre et des troupes coloniales

Ajoutons enfin que, sur le plan culturel, malgré l'indéniable réussite du mouvement de rajeunissement et de modernisation de la littérature bretonnante, dans les années vingt, incarné par la revue Gwalarn lancée en 1928, et malgré la volonté de retrouver un enracinement proprement celte dans les arts plastiques et décoratifs tout comme en architecture, le prestige de Paris comme foyer de créativité intellectuelle s'impose tant aux littérateurs qu'aux plasticiens bretons de l'entre-deux-guerres.

D'où l'exaspération des plus exaltés des tenants du séparatisme qui estiment que la majorité des Bretons est incapable de rejeter l'hégémonie 
arrogante et insidieuse imposée par Paris, que tous ces gens ont perdu leur âme avec leur langue et que donc, seule l'action continue et volontariste d'une minorité pourra provoquer le sursaut nécessaire. Pour cela, il faut exploiter toutes les occasions d'affaiblissement de cette hégémonie. C'est dire l'espoir que provoqua la " divine surprise " de l'écrasante défaite de juin 1940, qu'ils interprétèrent comme la confirmation de la justesse et de la légitimité de leurs analyses. Ils vont donc jouer la carte allemande et certains vont aller très loin dans l'espoir d'arracher à l'occupant une adhésion que ce dernier refuse, préférant jouer avec Vichy le scénario de la collaboration contre ceux qui prônent la Résistance à l'occupation; or Pétain condamne toute tentative séparatiste qui s'ajouterait aux amputations territoriales imposées par le vainqueur. Finalement le rôle des formations paramilitaires issues du PNB dans la traque des résistants va disqualifier tout projet, même simplement d'autonomie, pour plusieurs décennies.

En résumé, ce premier vingtième siècle n'a fait que renforcer la francisation d'une Bretagne devenue partie prenante de tous les objectifs communs du destin collectif de la Nation : récupération de l'Alsace-Lorraine et coup d'arrêt aux ambitions territoriales de l'Allemagne Wilhelmienne puis, relance d'une politique de grande puissance appuyée sur un Empire colonial qui a pu résister au choc d'une guerre mondiale et que la victoire a encore agrandi, qu'il faut donc préserver tout en développant ses potentialités économiques. Comme ailleurs en France, la guerre a contribué à accroître l'emprise de l'État déjà pourvoyeur d'emplois, mais désormais assistant, tout particulièrement, la veuve et l'orphelin et tous les anciens combattants frappés dans leur chair par les mutilations et les séquelles de leurs blessures et maladies. Tout ceci accroît l'exaspération d'une poignée de militants, partisans de la manière forte, et décidés à sauver la nation bretonne menacée d'être totalement absorbée par une République dont ils constatent la relative impuissance politique. Ils estiment même qu'elle est condamnée à brève échéance par le dynamisme des régimes autoritaires et totalitaires qui ne peuvent que l'emporter dans leur confrontation avec des démocraties impuissantes et, par nature, engluées dans les principes et les scrupules d'un autre âge. 